\title{
Assessment of the cardiac safety and pharmacokinetics of a short course, twice daily dose of orally-administered mifepristone in healthy male subjects
}

\author{
Borje Darpo ${ }^{1,2}$, Roy Bullingham ${ }^{3}$, Daniel L. Combs ${ }^{4}$, Georg Ferber ${ }^{5}$, Karen Hafez ${ }^{6}$ \\ ${ }^{1}$ Department of Clinical Sciences, Danderyd's Hospital, Division of Cardiovascular Medicine, Stockholm, Sweden \\ ${ }^{2}$ iCardiac Technologies, Rochester, NY, USA \\ ${ }^{3}$ Consultant Milford, NJ, USA \\ ${ }^{4}$ Combs Consulting Service, Mountain View, CA, USA \\ ${ }^{5}$ Statistik Georg Ferber GmbH, Riehen, Switzerland \\ ${ }^{6}$ Corcept Therapeutics, Inc., Menlo Park, CA, USA
}

\begin{abstract}
Background: Mifepristone is approved to control hyperglycemia in adults with endogenous Cushing's syndrome and is described as a mildly QTc prolonging drug, based on a TQT study. The aim of the present study was to assess the effect of mifepristone on the QTc interval at plasma mifepristone concentrations exceeding those observed in the TQT study.

Methods: Twenty healthy, male volunteers were given three doses of $1200 \mathrm{mg}$ mifepristone every $12 \mathrm{~h}$ with a high-fat meal in a randomized, placebo-controlled 2-period crossover study. Holter ECG recordings were made on Day 1 and 2.

Results: Eighteen subjects completed the study. Mean peak plasma mifepristone concentrations were $4.01 \mu \mathrm{g} / \mathrm{mL}$ (CV: 31\%) on the first dose and $5.77 \mu \mathrm{g} / \mathrm{mL}$ (CV: 29\%) on the third dose. Mifepristone did not have a meaningful QTc effect. The placebo-corrected, change-from-baseline $Q T c F(\triangle \triangle Q T c F)$ was between -1.6 and $0.7 \mathrm{~ms}$ on the first dose (upper bound of $90 \%$ CI $3.8 \mathrm{~ms}$ ) and the largest $\triangle \triangle Q T c F$ on the third dose was $4.9 \mathrm{~ms}$ (upper bound of $90 \%$ CI: $8.4 \mathrm{~ms}$ ). Concentration effect modeling showed a slightly negative slope of $-0.01 \mathrm{~ms} / \mathrm{ng} / \mathrm{mL}$.

Conclusions: Mifepristone did not cause a clinically meaningful QTc prolongation in healthy volunteers at plasma concent rations of mifepristone and its main metabolites that clearly exceeded those seen in a previous TQT study. (Cardiol J 2013; 20, 2: 152-160)
\end{abstract}

Key words: mifepristone, QT/QTc, TQT study, PK, PK/PD, early QT assessment

\section{Introduction}

Mifepristone $\left(\right.$ Korlym $\left.^{\mathrm{TM}}\right)$ is an antagonist of the type II glucocorticoid receptor (GR-II) and progesterone receptor approved with orphan-drug status as a once-daily oral medicine to control hyperglycemia in adult patients with endogenous Cushing's syndrome who have failed surgery or who are not candidates for surgery [1]. Patients initiate mifepristone treatment at a dose of $300 \mathrm{mg} /$ day administered with

Address for correspondence: Borje Darpo, MD, PhD, Karolinska Institutet, Department of Clinical Sciences,

Danderyd's Hospital, Division of Cardiovascular Medicine, Stockholm, Danderyds Hospital, SE 18288 Danderyd, Sweden, tel: +46 763902 130, e-mail: borje.darpo@telia.com 
food. The dose can then be titrated by $300 \mathrm{mg}$ increments up to $1200 \mathrm{mg}$ daily by assessing tolerability and degree of improvement [2].

Reported mifepristone pharmacokinetics are largely from studies using relatively low doses given as a single dose or for only a few days [3, 4]. Over the whole dose range now studied, from 2 to $1800 \mathrm{mg}$, mifepristone reveals complex pharmacokinetics that at higher doses are not linear or dose proportional and are time-dependent. These features arise from its exclusive and extensive CYP3A metabolism, strong CYP3A inhibition leading to autoinhibited metabolism, and CYP3A autoinduction. Additionally, there may be some role for alpha acid glycoprotein binding at low doses and an absorption limit at high doses. There is also a dose dependent food effect that varies from none to greater than $50 \%$ increase in exposure for multiple doses from $300 \mathrm{mg}$ and $1200 \mathrm{mg}$, respectively. The mean terminal half-life is long ( 2 to 4 days after multiple dosing). There are 3 major metabolites that have pharmacodynamic activities similar to, but less potent, than that of mifepristone. Designing a QT study for a drug with this constellation of properties is an interesting challenge.

Current labeling for mifepristone for Cushing's syndrome warns against use with QT interval-prolonging drugs, or in patients with potassium channel variants resulting in a long QT interval [1]. This advice is based on the results of a parallel group thorough QT (TQT) assessment of therapeutic (600 mg OD) and supratherapeutic (1800 OD) doses of mifepristone administered under fasting conditions for 14 days. The $1800 \mathrm{mg}$ but not the $600 \mathrm{mg}$ dose caused a small mean QTc prolongation (placebo corrected change from baseline) of 3 to $7 \mathrm{~ms}$ between 6 and $20 \mathrm{~h}$ post-dosing on Day 7 of dosing. No time-point had a $90 \%$ upper confidence interval (CI) that exceeded $11 \mathrm{~ms}$ (data on file). In a concentration-response analysis, no PK/QTc relationship was identified, but the dynamic range of plasma mifepristone concentrations was small at steady state. The study had a large number of subject dropouts, which complicated the data interpretation.

The rationale for the current study was to conduct a QT study in which higher plasma mifepristone concentrations than those observed in the TQT study were projected with shorter study duration in order to avoid CYP3A autoinduction. To achieve this, $1200 \mathrm{mg}$ doses of mifepristone were given with food using a short course (every $12 \mathrm{~h}$ for 3 doses) placebo-controlled crossover study.

\section{Methods}

\section{Subjects}

The study enrolled healthy non-smoking male volunteers without significant medical history aged $18-45$ years with body mass indices (BMI) between 19 and $32 \mathrm{~kg} / \mathrm{m}^{2}$. Physical examinations, 12-lead ECGs, and clinical laboratory evaluations were performed within 30 days prior to dosing. Corrected QT intervals $(\mathrm{QTcF}) \leq 450 \mathrm{~ms}$ were required at screening. If appropriate, two approved forms of contraception were used by female partners of the male subjects for the duration of the study. Ingestion of citrus and quinine was avoided by subjects during the study. Except for paracetamol, over-the counter or prescription medications were not allowed within 30 days prior to first study dose or during the study.

All volunteers gave written informed consent prior to any study related procedures. The study was approved by an independent Ethics Committee (Plymouth Independent Ethics Committee) and conducted in accordance with the Declaration of Helsinki and Good Clinical Practice guidelines as set forth by the International Conference on Harmonisation and the U.S. Code of Federal Regulations.

\section{Study design}

The study was randomized, double blind and placebo-controlled, and used a 2-way crossover design performed at a single clinical site. Subjects were randomized as they completed screening assessments $1: 1$ to 1 of 2 treatment sequences. There was a 2 -week washout period between periods.

In each period, subjects received either oral mifepristone $1200 \mathrm{mg}$ or matching placebo in a double-blinded fashion every $12 \mathrm{~h}$ for 3 doses, on the morning and evening of Day 1 and morning of Day 2. Each treatment was administered within $30 \pm 15$ min of a high fat (50\% fat) meal with room temperature water. Participants were confined to the clinical centre on Days 1-3 of each dosing period and returned for the end-of-study visit.

\section{ECG recordings}

Electrocardiograms (ECG) were obtained digitally using a continuous 12-lead Holter recorder (Global Instrumentation ${ }^{\circledR}$ M12R, Buffalo, NY). The recording started $1 \mathrm{~h}$ before dosing on Day 1 and continued until $24 \mathrm{~h}$ after dosing on Day 2. Recordings were stored on electronic media and were 
shipped to the central ECG laboratory (iCardiac Technologies, Rochester, NY) after each dosing cohort. ECGs were extracted from the continuous recordings at the same time points as blood draws (see below). Subjects rested in the semi-recumbent or supine position for at least $10 \mathrm{~min}$ before and 5 min after each of these time points. Using the TQTPlus $^{\circledR}$ Technique (iCardiac Technologies, Rochester, NY), 10-s digital 12-lead ECG tracings were extracted from the continuous recordings using criteria for signal-to-noise ratio and stability of heart rate (HR). Ten replicate ECGs were extracted in close succession within each extraction window. QT interval measurements were performed using the High Precision QT Analysis (HPQT) technique, which utilises the COMPAS ${ }^{\circledR}$ software for interval measurements. All recorded cardiac beats in all replicates were assessed for quality, and signal-to-noise ratios were categorized as of "high" or "low" confidence. All "high confidence" beats were accepted into the analysis without manual adjustment, whereas all "low confidence" beats were fully reviewed manually and adjudicated using pass-fail criteria [5]. Final quality assessment was performed by a board certified cardiologist. Review of ECGs from a particular subject was performed by a single reader and baseline and on-treatment ECGs measurements in a subject were based on the same lead. For PR and QRS intervals and T-wave morphology, 3 of the 10 ECG replicates with the highest signal to noise ratio were selected for review. The median $\mathrm{QT}$ and $\mathrm{RR}$ value from each of the 10 extracted replicates was calculated and the mean of all available medians from a nominal time point was used as the subject's reportable value at that time point.

\section{Pharmacokinetic sampling}

Serial 12-h mifepristone and active metabolite plasma concentration profiles were collected within 30 min prior to dosing and at $0.5,1,1.5,2$, $3,4,5,6,7,8,10$, and $12 \mathrm{~h}$ after Dose 1 (Day 1 ) and Dose 3 (Day 2) of both periods. An additional blood sample was taken on Day 3, $24 \mathrm{~h}$ after the last dose of medication. Total plasma concentrations of mifepristone and the 3 active metabolites RU 42633 (mono-demethylatedmetabolite), RU 42698 (hydroxylated metabolite) and RU 42848 (di-demethylatedmetabolite) were determined by MicroConstants (San Diego, California) using a validated liquid chromatography assay method with tandem mass spectrometry (LC/MS/MS). The limits of quantitation were $10 \mathrm{ng} / \mathrm{mL}$ for each analyte.

\section{Safety determinations}

Safety assessments included measurement of vital signs (blood pressure, HR, respiratory rate, and oral temperature), 12-lead ECGs, clinical laboratory tests, and adverse event monitoring.

\section{Analyses and statistics}

Based on the TQT study where an $1800 \mathrm{mg}$ mifepristone dose caused a small mean QTc prolongation (placebo corrected change from baseline) of 3 to $7 \mathrm{~ms}$ between 6 and $20 \mathrm{~h}$ post dosing on Day 7 of dosing, the study was calculated to have $80 \%$ power to exclude an effect of $15 \mathrm{~ms}$ at all time points on Day 2 with 16 subjects assuming standard deviation of the change from baseline QTc of $7 \mathrm{~ms}$ and independence between the tests at different time points. Data from all randomised subjects were included in the ECG analyses. Safety analyses included all subjects receiving study treatments. Pharmacokinetic analyses were performed on all subjects treated with mifepristone from whom at least one post dose sample above the limit of quantitation was obtained. Statistical reporting was performed using $\mathrm{R}$ for Windows (v2.13.0).

ECG analyses. Descriptive statistics (e.g., frequency, percent, mean, standard deviation [SD], coefficient of variation [CV\%], median, maximum and minimum) were used to summarize the QTc, and other ECG variables (HR, PR, RR, and QRS intervals), and corresponding changes from baseline. The primary endpoint was QTcF (= QT/ $/ \mathrm{RR}^{0.33}$ ) [6]. For each time point, a linear mixed effects model was fitted with QTcF as dependent variable, sequence, period, and treatment as fixed effects, baseline as covariate and subject (intercept) as random effect. A 2 -sided $90 \%$ CI was calculated for the contrasts "mifepristone - placebo" $\Delta \mathrm{QTcF}(\Delta \Delta \mathrm{QTcF})$.

Analysis of QTc outliers (QTcF > $450 \mathrm{~ms}$, $>480 \mathrm{~ms}$ and $>500 \mathrm{~ms}$ and $\Delta \mathrm{QTcF}>30 \mathrm{~ms}$ and $>60 \mathrm{~ms}$ ) and treatment emergent changes of T-wave morphology was performed.

Pharmacokinetic analyses. Maximum peak concentration (Cmax), time to maximum concentration (Tmax), and the area under the concentration-time curve from $0 \mathrm{~h}$ to $12 \mathrm{~h}\left(\mathrm{AUC}_{0-12}\right.$ computed using the linear trapezoidal rule) were derived from the plasma concentration profiles for mifepristone and its metabolites after dose 1 and dose 3 . Standard non-compartmental computation methods were used (WinNonlin ${ }^{\circledR}$ Professional version 5.2, Pharsight, St. Louis, MO). Summary statistics included count, mean, median, SD, minimum, maximum, $\mathrm{CV} \%$ and a 2 -sided $90 \% \mathrm{CI}$. 
Pharmacokinetic/pharmacodynamic analyses. The relationship between plasma concentration of mifepristone and metabolites and $\Delta \Delta \mathrm{QTcF}$ was investigated using a linear mixed-effect model:

$$
\Delta \Delta Q \mathrm{TcF}_{\mathrm{ij}}=\text { Intercept }_{\mathrm{i}}+\text { Slope }_{\mathrm{i}} \times \text { Conc }_{\mathrm{ij}}+\varepsilon_{\mathrm{ij}}
$$

where $\Delta \Delta \mathrm{QTCF}_{\mathrm{ij}}$ was the time-matched, placebo-corrected change-from-baseline QTcF for subject $i$ at time $j$ with mifepristone or its main metabolites concentration Conc $_{\mathrm{ij}}$. Time matched concentration was included in the model as a variable and subjects as a random effect for both intercept and slope, whenever applicable. The residual $\varepsilon_{\mathrm{ij}}$ was assumed to be identical, independent, normally distributed with mean 0 and variance $\sigma^{2}$. Three models were used where Model 1 used a fixed and random intercept, Model 2 set the fixed intercept to 0 but allowed for a random intercept and Model 3 had no intercept.

A plot of standardized residuals vs. fitted values was used to examine departure from model assumptions. In addition, normal Q-Q plots of the random effects and the within-subject errors were used to investigate the normality of the random effects and the within-subject errors, respectively. A final assessment of the adequacy of the linear mixed effects model was provided by a goodness-of-fit plot (i.e. the observed concentration quantile- $\Delta \Delta \mathrm{QTCF}$ plot) [7] to check both the assumption of linearity between the concentrations of mifepristone or its main metabolites and $\Delta \Delta \mathrm{QTCF}$ and how well the predicted $\Delta \Delta \mathrm{QTCF}$ matched the observed data in the regions of interest. The goodness-of-fit plot was generated by binning the independent variable of concentration into deciles. The mean $\Delta \Delta \mathrm{QTCF}$ with $90 \%$ CI within each decile was computed and plotted at the corresponding median concentration within the decile [7]. The model providing the best fit as judged from the diagnostic plots and the Akaike Information Criterion was to be selected.

\section{Results}

Twenty male subjects were randomized and 18 completed the study. Two subjects were discontinued, one due to and adverse event of skin rash and one due to elevated ALT. A third subject withdrew consent during the first dosing period, but reentered the study for the second dosing period. The majority of subjects were Caucasian (13/20, 65\%), 3 were Black/ /African American (15\%), 2 were Asian (10\%) and 2 were categorized as Other race (10\%). Mean \pm SD age was $31 \pm 4$ years and BMI was $25 \pm 3 \mathrm{~kg} / \mathrm{m}^{2}$.

\section{QTc analyses}

On both Day 1 and Day 2, the $\Delta$ QTcF diurnal pattern was similar during the placebo and the mifepristone treatments, with a shortening during the first $10 \mathrm{~h}$ post-dosing (Fig. 1). On Day 2, the $\Delta \mathrm{QTCF}$ shortening was somewhat larger on mifepristone than on placebo. Later during Day 2, $\triangle \mathrm{QTCF}$ was similar on both treatments, with the exception of the 24-h time point. At this time point, which occurred in the morning on Day $3, \Delta \mathrm{QTcF}$ was $-2.3 \mathrm{~ms}$ for placebo and $2.6 \mathrm{~ms}$ for mifepristone. The resulting mean placebo-corrected $\Delta \mathrm{QTcF}$ $(\Delta \Delta \mathrm{QTCF})$ was within a very narrow range on Day 1 , -1.6 to $0.7 \mathrm{~ms}$ (Fig. 2), and the upper bound of the $90 \% \mathrm{CI}$ was below $4 \mathrm{~ms}$ at all time points (Table 1). On Day 2, there was an initial shortening of the QTc interval with mean $\Delta \Delta \mathrm{QTCF}$ between -1.7 and $-5.2 \mathrm{~ms}$. Mean $\Delta \Delta \mathrm{QTcF}$ thereafter remained around $0 \mathrm{~ms}$ ( -1.5 to $0.8 \mathrm{~ms}$ ) between 5 and $12 \mathrm{~h}$ after dosing, whereas the 24 -h value (i.e., in the morning of Day 3) reached $4.9 \mathrm{~ms}$ (90\% CI 1.4-8.4 ms). The upper bound of the $90 \%$ CI was well below $10 \mathrm{~ms}$ at all time points (Table 1 ).

There were no subjects with absolute QTcF values exceeding $480 \mathrm{~ms}$ or $\Delta \mathrm{QTCF}$ exceeding $30 \mathrm{~ms}$. All subjects had QTcF values less than $450 \mathrm{~ms}$ at all time points except 1 subject with a QTcF value that exceeded $450 \mathrm{~ms}$ at the 24 -h time point of Day 2. The only observed T-wave abnormality was flattened T-waves, which was seen at 1 time point on placebo and 1 time point on mifepristone.

The mean SD of $\Delta \mathrm{QTc}$ across time points was below $7.0 \mathrm{~ms}$ for mifepristone on Day 1 and placebo on both days, whereas the precision was somewhat lower for mifepristone on Day 2 (mean of $7.8 \mathrm{~ms}$ ).

\section{Heart rate, $\mathrm{PR}$ and $\mathrm{QRS}$, ECG morphology analysis}

On both Day 1 and Day 2, change-from-baseline heart rate $(\Delta \mathrm{HR})$ was small at all time points for both placebo and mifepristone. On Day 1, placebo-corrected $\Delta \mathrm{HR}(\Delta \Delta \mathrm{HR})$ showed a slight lowering of the mean HR of up to $3.7 \mathrm{bpm}$ during the first $2 \mathrm{~h}$ after dosing, after which the HR was unchanged throughout the dosing interval. On Day 2, the same pattern was observed with a slight lowering of 2 to $3 \mathrm{bpm}$ immediately after the morning dose with somewhat higher values in the afternoon.

A small PR interval shortening was observed on both placebo and on mifepristone on Day 1 and on Day 2, with change-from-baseline PR $(\Delta \mathrm{PR})$ reaching -5 to $-6 \mathrm{~ms}$ (data not shown). The placebo-corrected $\Delta \mathrm{PR}(\Delta \Delta \mathrm{PR})$ varied between $-3.0 \mathrm{~ms}$ and $4.7 \mathrm{~ms}$ on Day 1 and $-3.7 \mathrm{~ms}$ and $2.1 \mathrm{~ms}$ on 


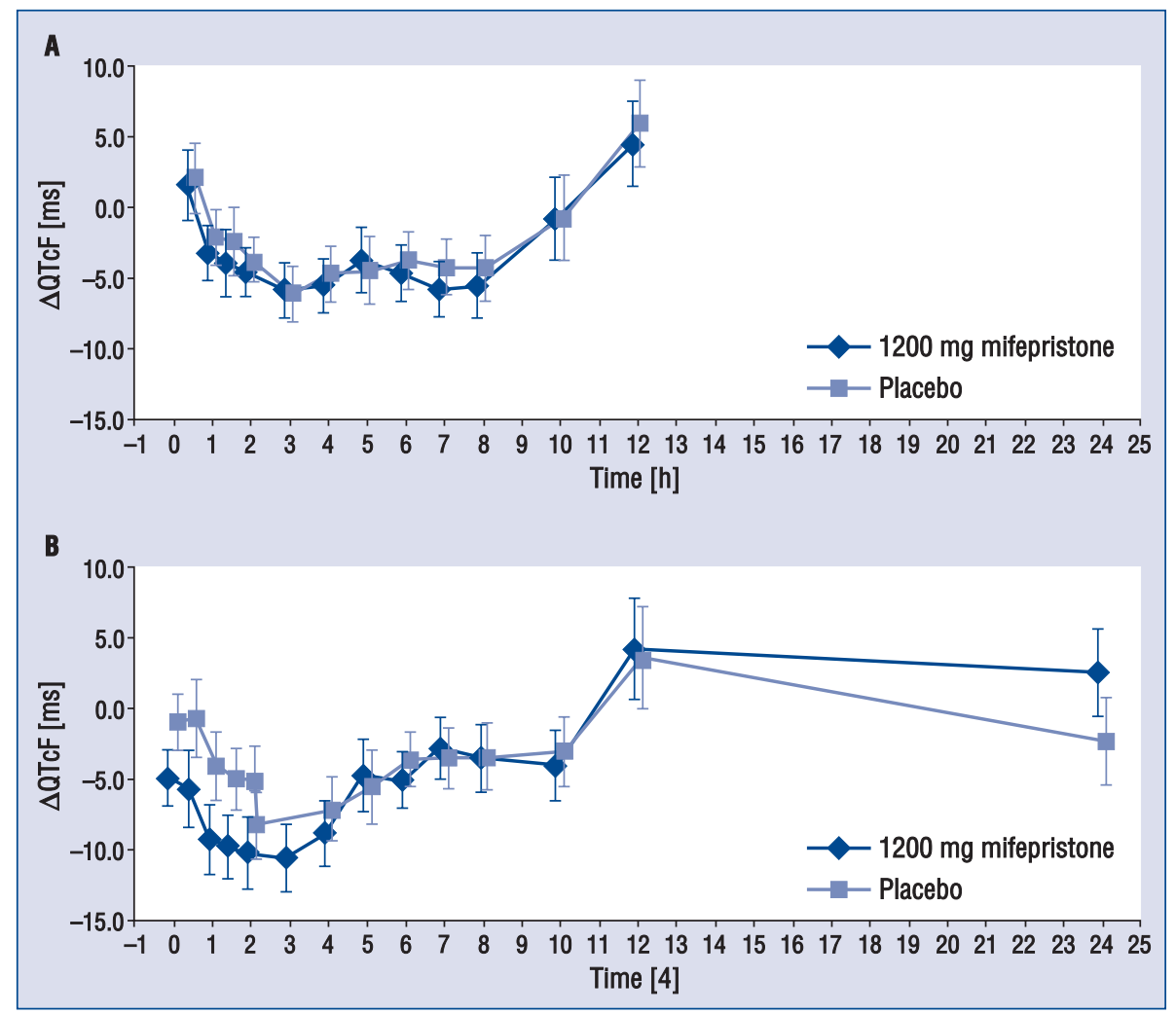

Figure 1. Change-from-baseline $\mathrm{QTcF}(\triangle \mathrm{QTCF}$, mean $\pm \mathrm{SE})$ on Day 1 (A) and Day 2 (B).

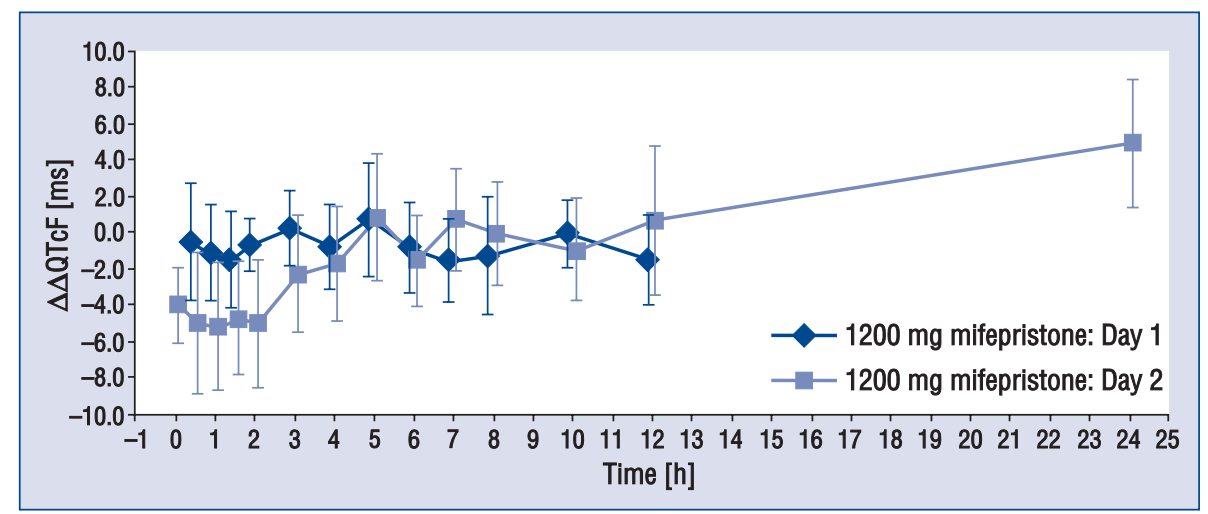

Figure 2. Placebo-corrected change-from-baseline QTcF ( $\Delta \Delta \mathrm{QTcF}$, mean with $90 \%$ confidence intervals) on Day 1 and Day 2.

Day 2. Mifepristone did not have an effect on QRS duration and $\Delta \Delta$ QRS was essentially unchanged with all values within $\pm 1.0 \mathrm{~ms}$.

\section{Pharmacokinetic parameters}

Cmax and $\mathrm{AUC}_{0-12}$ increased from dose 1 to dose 3 for both mifepristone and its metabolites (Table 2); the mean ratio for Cmax between dose
3 and dose 1 was 1.50 for mifepristone, 1.04 for RU 42633, 1.29 for RU 42698 and 1.39 for RU 42848. For $\mathrm{AUC}_{0-12}$ the accumulation between dose 1 and dose 3 was generally higher than for Cmax with ratios of 1.62, 1.10, 1.44 and 1.65 for mifepristone, RU 42633, RU 42698 and RU 42848, respectively. Overall, the accumulation observed for mifepristone, RU 42698 and RU 42848 was modest, and 
Table 1. Placebo-corrected changes from Day 1 redoes baseline $\mathrm{QTcF}(\Delta \Delta \mathrm{QTcF}, \mathrm{ms})$ across time points.

\begin{tabular}{|c|c|c|c|c|}
\hline \multirow[t]{2}{*}{ Time point [h] } & \multicolumn{2}{|c|}{ Day 1} & \multicolumn{2}{|c|}{ Day 2} \\
\hline & {$[\mathrm{ms}]$ mean $\pm \mathrm{SE}$} & $90 \% \mathrm{Cl}$ & {$[\mathrm{ms}]$ mean $\pm \mathrm{SE}$} & $90 \% \mathrm{Cl}$ \\
\hline 0 & NA & NA & $-4.0 \pm 1.3$ & -6.1 to -1.9 \\
\hline 0.5 & $-0.5 \pm 2.0$ & -3.8 to 2.7 & $-5.0 \pm 2.4$ & -8.9 to -1.1 \\
\hline 1 & $-1.2 \pm 1.6$ & -3.8 to 1.4 & $-5.2 \pm 2.1$ & -8.7 to -1.7 \\
\hline 1.5 & $-1.5 \pm 1.6$ & -4.2 to 1.2 & $-4.8 \pm 1.9$ & -7.9 to 1.7 \\
\hline 2 & $-0.7 \pm 0.9$ & -2.2 to 0.7 & $-5.0 \pm 2.1$ & -8.6 to -1.5 \\
\hline 3 & $0.2 \pm 1.2$ & -1.8 to 2.2 & $-2.3 \pm 2.0$ & -5.5 to 0.9 \\
\hline 4 & $-0.8 \pm 1.4$ & -3.2 to 1.5 & $-1.7 \pm 1.9$ & -4.9 to 1.5 \\
\hline 5 & $0.7 \pm 1.9$ & -2.4 to 3.8 & $0.8 \pm 2.1$ & -2.7 to 4.3 \\
\hline 6 & $-0.8 \pm 1.5$ & -3.4 to 1.7 & $-1.5 \pm 1.5$ & -4.0 to 1.0 \\
\hline 7 & $-1.6 \pm 1.4$ & -3.9 to 0.8 & $0.7 \pm 1.7$ & -2.1 to 3.5 \\
\hline 8 & $-1.3 \pm 2.0$ & -4.5 to 1.9 & $-0.1 \pm 1.7$ & -2.9 to 2.8 \\
\hline 10 & $-0.1 \pm 1.1$ & -1.9 to 1.8 & $-1.0 \pm 1.7$ & -3.8 to 1.8 \\
\hline 12 & $-1.5 \pm 1.5$ & -4.0 to 0.9 & $0.6 \pm 2.5$ & -3.5 to 4.8 \\
\hline 24 & & & $4.9 \pm 2.1$ & 1.4 to 8.4 \\
\hline
\end{tabular}

Table 2. Pharmacokinetic parameters (mean; CV\%) of mifepristone and metabolites.

\begin{tabular}{|c|c|c|c|c|c|c|}
\hline Analyte & Day & & $\operatorname{Tmax}[\mathrm{h}]$ & $\mathrm{Cmax}[\mu \mathrm{g} / \mathrm{mL}]$ & Ctrough $[\mu \mathrm{g} / \mathrm{mL}]$ & $\mathrm{AUC}_{\tau}[\mathrm{h} \times \mu \mathrm{g} / \mathrm{mL}]$ \\
\hline \multirow[t]{6}{*}{ Mifepristone } & 1 & $\mathrm{~N}$ & 20 & 20 & 19 & 20 \\
\hline & & Mean & 4.37 & 4.01 & 2.05 & 30.9 \\
\hline & & CV\% & 43.8 & 30.6 & 35.0 & 30.5 \\
\hline & 2 & $\mathrm{~N}$ & 18 & 18 & 18 & 18 \\
\hline & & Mean & 5.13 & 5.77 & 3.70 & 49.0 \\
\hline & & CV\% & 55.1 & 28.8 & 41.5 & 28.6 \\
\hline \multirow[t]{6}{*}{ RU 42633} & 1 & $\mathrm{~N}$ & 20 & 20 & 19 & 20 \\
\hline & & Mean & 6.70 & 3.02 & 2.60 & 27.2 \\
\hline & & CV\% & 37.7 & 41.7 & 32.5 & 31.2 \\
\hline & 2 & $\mathrm{~N}$ & 18 & 18 & 18 & 18 \\
\hline & & Mean & 8.21 & 2.93 & 2.57 & 28.9 \\
\hline & & CV\% & 47.8 & 31.1 & 34.9 & 29.2 \\
\hline \multirow[t]{6}{*}{ RU 42698} & 1 & $\mathrm{~N}$ & 20 & 20 & 19 & 19 \\
\hline & & Mean & 10.36 & 0.78 & 0.74 & 6.45 \\
\hline & & CV\% & 25.3 & 34.4 & 39.8 & 33.2 \\
\hline & 2 & $\mathrm{~N}$ & 18 & 18 & 18 & 18 \\
\hline & & Mean & 7.24 & 0.97 & 0.88 & 9.26 \\
\hline & & CV\% & 69.1 & 34.5 & 38.3 & 31.3 \\
\hline \multirow[t]{6}{*}{ RU 42848} & 1 & $\mathrm{~N}$ & 20 & 20 & 19 & 20 \\
\hline & & Mean & 10.41 & 1.51 & 1.44 & 12.1 \\
\hline & & CV\% & 20.3 & 35.3 & 29.4 & 28.9 \\
\hline & 2 & $\mathrm{~N}$ & 18 & 18 & 18 & 18 \\
\hline & & Mean & 8.99 & 1.96 & 1.82 & 19.2 \\
\hline & & CV\% & 37.1 & 33.3 & 36.2 & 26.8 \\
\hline
\end{tabular}


RU 42633 had little, if any, accumulation. Tmax for mifepristone changed little between dose 1 ( $4.4 \mathrm{~h}$; CV 44\%) and dose 2 (5.1 h; CV 55\%).

\section{Pharmacokinetic/pharmacodynamic relationships}

Model 2 with mean intercept fixed to 0 (with variability) was found to fit the data best, i.e., provided the best fit as judged from the diagnostic plots and the Akaike Information Criterion among the 3 candidate models and was therefore chosen for the analysis.

Given the long half-life of mifepristone and its metabolites, analysis of the predose sample on Day 1 in period 2 from 9 subjects who were treated with the sequence mifepristone $\rightarrow$ placebo was performed. In $7 / 9$ of these subjects, low but quantifiable levels of mifepristone (mean $0.51 \pm$ $\pm 0.34 \mu \mathrm{g} / \mathrm{mL}$ ) and of its metabolites were detected. Therefore, data from only the 10 subjects who received placebo in period 1 and mifepristone in period 2 was used in the primary PK-QTc analysis. For the secondary PK-QTc analysis, data from all 20 subjects were used.

Concentration effect modeling demonstrated a slightly inverse relation between mifepristone plasma concentrations and $\Delta \Delta \mathrm{QTcF}$ with a negative slope of $-0.0010 \mathrm{~ms} / \mathrm{ng} / \mathrm{mL}$ (CI: -0.0014 to -0.0005 ; $\mathrm{p}=0.0004$ ) in the primary analysis (that included only subjects dosed in sequence placebo - mifepristone) and a non-significant slope of $-0.0010 \mathrm{~ms} /$ $/ \mathrm{ng} / \mathrm{mL}$ (CI: -0.0026 to $0.0005 ; \mathrm{p}=0.2731$ ) in the secondary analysis, which included all 20 subjects. The goodness-of-fit plot (Fig. 3) shows the mean $\Delta \Delta \mathrm{QTcF}(90 \% \mathrm{CI})$ within each mifepristone plasma concentration decile and the model-predicted mean $\Delta \Delta \mathrm{QTcF}$ with $90 \% \mathrm{CI}$. The predicted $\Delta \Delta \mathrm{QTcF}$ at the observed mean peak plasma concentration of $5.77 \mu \mathrm{g} / \mathrm{mL}$ was $-5.7 \mathrm{~ms}$, which is consistent with the results of the time-matched analysis.

None of the 3 major metabolites of mifepristone were associated with a concentration dependent prolongation of $\Delta \Delta \mathrm{QTcF}$ (data not shown).

\section{Tolerability}

Fifteen (75\%) subjects experienced 44 treatment emergent adverse events during the mifepristone periods and 6/20 (30\%) experienced 11 events during the placebo periods. The majority of adverse events were mild in intensity (39/44, $89 \%$ in the mifepristone periods and $9 / 11,81 \%$ in the placebo periods). The remainder of events was of moderate intensity. There were no serious adverse events. Common events (occurring in $\geq 3$ subjects) during the mifepristone periods were

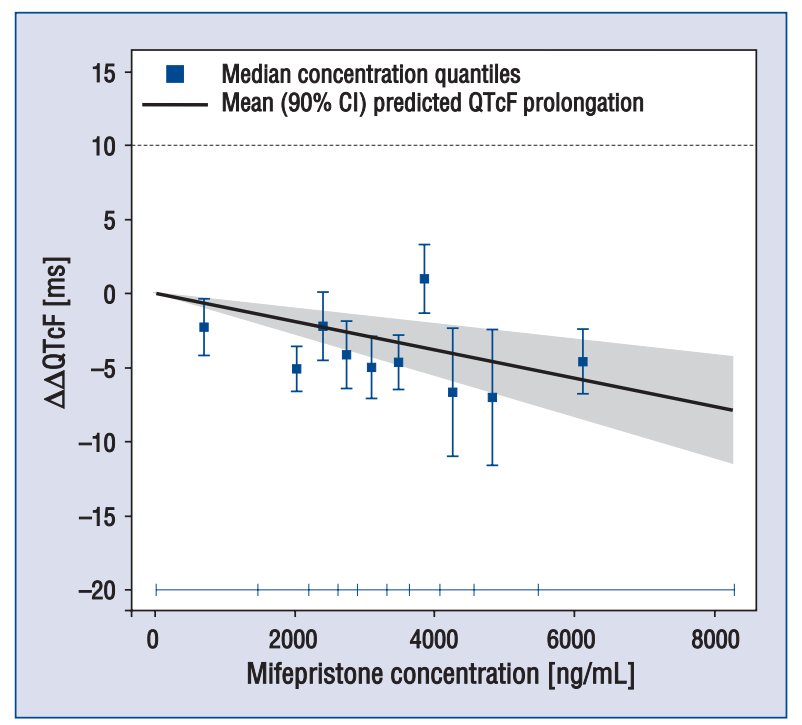

Figure 3. Observed and predicted relation between mifepristone plasma levels and $\Delta \Delta \mathrm{QT} c \mathrm{~F}$. Primary analysis in 10 subjects from dosing sequence Placebo $\rightarrow$ Mifepristone. Blue vertical bars show the observed mean $\Delta \Delta \mathrm{QTcF}$ with $90 \%$ confidence interval $(\mathrm{Cl})$ within each plasma concentration decile. The solid black line with gray shaded area represents the model-predicted mean $\Delta \Delta \mathrm{QTcF}$ with $90 \% \mathrm{Cl}$. The horizontal blue lines with notches show the range of plasma concentrations within each decile.

abdominal cramps, dry mouth, headache, insomnia, dizziness, and rash.

There were no clinically significant changes in clinical laboratory measurements, vital signs, ECG safety parameters, or physical findings other than the 1 subject withdrawn due to elevated ALT. The subject had an elevated ALT of $159.5 \mathrm{IU} / \mathrm{L}$ considered clinically significant. Upon retesting $24 \mathrm{~h}$ later, ALT level remained elevated and the subject was withdrawn. ALT level was within normal range upon retesting 1 week later.

\section{Discussion}

Mifepristone was recently approved in the US with the indication to control hyperglycemia in adult patients with endogenous Cushing's syndrome who have failed surgery or who are not candidates for surgery [8]. The label states that mifepristone prolongs the QT interval in a doserelated manner and includes cautionary statements that are based on the results of a previous multiple dose TQT study conducted over 14 days.

The current study was designed to assess the ECG effects of mifepristone at plasma drug con- 
centrations that clearly would exceed those seen in patients on chronic dosing with the maximum recommended dose $1200 \mathrm{mg}$ once daily. Mifepristone has complex metabolism that leads to pharmacokinetics that are not linear or dose-proportional, and are time dependent. Even though the mean terminal half-life of the drug after multiple dosing is long ( 2 to 4 days in healthy subjects), drug exposure at steady state is similar to that of the first dose. To overcome the increased clearance of the drug with multiple dosing and obtain supratherapeutic plasma drug concentrations, a short-term dosing schedule was used with 3 doses of $1200 \mathrm{mg}$ administered every $12 \mathrm{~h}$ with serial ECG assessment on 2 consecutive days. Since food increases mifepristone exposure, the drug was administered with a high-fat meal. Mean mifepristone Cmax reached $4.01 \mu \mathrm{g} / \mathrm{mL}$ after the first dose on Day 1 and $5.77 \mu \mathrm{g} / \mathrm{mL}$ after the third dose on Day 2, compared to that of $3.92 \pm 1.37 \mu \mathrm{g} / \mathrm{mL}$ (95\% CI 3.56-4.28) at steady-state for fasted subjects given an $1800 \mathrm{mg}$ dose. Therefore, the dosing strategy of a short course with food provided exposures comparable to and higher than those of the TQT study at steady state with the first and third doses respectively.

To increase the power of the study to exclude small QTc effects, a 2-way crossover design was chosen with placebo and mifepristone in separate treatment periods. The study did not fulfill standard criteria for a TQT study as it did not include a positive control, but otherwise incorporated all TQT design-elements including strict control of experimental conditions and serial ECG recordings to capture effects observed at $\mathrm{Cmax}$ of both parent and major metabolites [9]. A high-precision QT measurement technique was used to increase the power of the assessment further. The achieved precision, measured as the SD of $\Delta \mathrm{QTcF}$, confirmed this approach: the mean SD of $\Delta \mathrm{QTcF}$ of 7.0 to $7.8 \mathrm{~ms}$ for both mifepristone and placebo compares favorably with other 'manually overseen' highly precise technologies, such as Eclysis [10], and is better than the precision typically achieved with standard semi-automated methods [5]. The results of the QT assessment were solidly negative in terms of the study's ability to exclude a QTcF effect exceeding $10 \mathrm{~ms}$ with the E14-defined time-matched analysis $[11,12]$. The results of the concentration-effect analysis were consistent with the time-matched analysis and demonstrated a reverse relation between mifepristone plasma concentrations and $\Delta \Delta \mathrm{QTCF}$ with a negative slope of $-0.0010 \mathrm{~ms} / \mathrm{ng} / \mathrm{mL}$ (CI: -0.0014 to -0.0005 ; $\mathrm{p}=0.0004)$. With a relatively small sample size of
20 subjects, it was thereby possible to exclude that plasma mifepristone concentrations exceeding those seen in the previous TQT have an effect on cardiac repolarization that would be of clinical concern.

These findings are in contrast to the results of an earlier parallel group TQT study in healthy subjects, conducted with 2 doses of mifepristone (a therapeutic $600 \mathrm{mg}$ dose and a supratherapeutic $1800 \mathrm{mg}$ dose) and placebo. On Day 7, mifepristone $1800 \mathrm{mg}$ OD caused a small QTc prolongation of 3 to $7 \mathrm{~ms}(\Delta \Delta \mathrm{QTCI})$; the upper bound of the $90 \%$ $\mathrm{CI}$ did not exceed $11 \mathrm{~ms}$ at any time point. On the same day, the mean $\Delta \Delta \mathrm{QTCI}$ in the mifepristone $600 \mathrm{mg}$ group was below $5 \mathrm{~ms}$ with all upper bounds of the CI below $10 \mathrm{~ms}$. Concentration-effect analysis showed no correlation of the observed QTc prolongation to either parent or any of the metabolites alone. Based on the TQT study, mifepristone seemed to cause a mild QTc prolongation with chronic dosing, with an apparent dose-response but unrelated to drug exposure. QTc effect with chronic dosing may therefore have a different underlying mechanism than direct inhibition of the hERG channel by the drug or its metabolites. Two potential mechanisms could be hypothesized, even though firm data supporting either one are lacking: an effect on hERG protein trafficking and/ /or an indirect pharmacodynamic effect.

Some drugs inhibit the transport of hERG proteins, or components thereof, from the endoplasmic reticulum to the cell membrane; this results in a reduction of the number of functional hERG channels at the cell surface, which may lead to QT prolongation. QT prolongation via this mechanism is not typically seen acutely but after some days of treatment. Examples of drugs that cause QT prolongation thorough inhibition of hERG trafficking are arsenic trioxide, pentamidine, and fluoxetine [13-18].The second and perhaps more likely mechanism is that mifepristone causes QT prolongation through an indirect mechanism. Mifepristone blocks the cortisol receptor and this leads to high circulating cortisol concentrations through feedback on the hypothalamic-pituitary axis. Elevated cortisol levels may activate mineralocorticoid receptors and this can lead to cellular potassium loss, which as such can result in prolongation of the QT interval. Small perturbation of the potassium balance in the myocardial cells may not be apparent from sampling in peripheral blood and may have contributed to the mild QT effect observed on chronic dosing.

The discrepancy between the present short-term study and the earlier TQT study with chronic 
dosing cannot be definitively explained without further studies. QT prolongation may be multifactorial and not always directly related to plasma concentrations of a drug or its metabolites. Furthermore, using single doses of drug to determine QTc effect may still in some cases leave uncertainty about the QTc effect of chronic dosing. Nonetheless, by combining concentration-effect modeling, an efficient design and a high precision QT measurement technique, a QTc effect exceeding $10 \mathrm{~ms}$ could be confidently excluded despite a relatively small sample size, which was substantially smaller than in most TQT studies [9]. Mifepristone has complex metabolism and it is difficult to achieve supratherapeutic exposure with chronic dosing. This study therefore also illustrates how a tailored approach based on known PK profile of a drug can results in high plasma levels despite somewhat unusual circumstances: in this case, a short-term frequent dosing schedule with high doses of the drug administered twice as often as intended in clinical practice and with a high-fat meal.

\section{Acknowledgments}

Support for conduct of the study and for medical writing was provided by Corcept Therapeutics, Inc. Medical writing and editing support provided by Patrice C. Ferriola, PhD.

Conflict of interest: The authors declare the following competing interests: No support from any organisation for the submitted work; BD consults for and owns stock in iCardiac Technologies; BD, RB, GF and DC have received consultancy fees from Corcept Therapeutics, Inc. in the previous 3 years; $\mathrm{KH}$ is an employee and holds stock/stock options of Corcept Therapeutics; no other relationships or activities that could appear to have influenced the submitted work.

\section{References}

1. Korlym prescribing information. Available at: http://www.accessdata.fda.gov/drugsatfda_docs/label/2012/202107s0001bl.pdf. Accessed December 2012
2. Fleseriu M, Biller BM, Findling JW, Molitch ME, Schteingart DE, Gross C. Mifepristone, a glucocorticoid receptor antagonist, produces clinical and metabolic benefits in patients with Cushing's syndrome. J Clin Endocrinol Metab, 2012; 97: 2039-2049.

3. Heikinheimo O, Lahteenmaki PL, Koivunen E et al. Metabolism and serum binding of RU 486 in women after various single doses. Hum Reprod, 1987; 2: 379-385.

4. Heikinheimo O. Clinical pharmacokinetics of mifepristone. Clin Pharmacokinet, 1997; 33: 7-17.

5. Darpo B, Fossa AA, Couderc JP et al. Improving the precision of QT measurements. Cardiol J, 2011; 18: 401-410.

6. Fridericia LS. Die Systolendauer im Elektrokardiogramm bei normalen Menchen und bei Herzkranken. Acta Med Scand, 1920; 53: 469-486.

7. Tornoe CW, Garnett CE, Wang Y, Florian J, Li M, Gobburu JV. Creation of a knowledge management system for QT analyses. J Clin Pharmacol, 2011; 51: 1035-1042.

8. Castinetti F, Brue T, Conte-Devolx B. The use of the glucocorticoid receptor antagonist mifepristone in Cushing's syndrome. Curr Opin Endocrinol Diabetes Obes, 2012; 19: 295-299.

9. Darpo B. The thorough QT/QTc study 4 years after the implementation of the ICH E14 guidance. Br J Pharmacol, 2010; 159: 49-57.

10. Dalen P, Vik T, Alverlind S, Jostell KG, Hardemark HG. Evaluation of the effects of AZD3480 on cardiac repolarization: A thorough QT/QTc study using moxifloxacin as a positive control. Clin Pharmacol Ther, 2010; 88: 532-539.

11. ICH Harmonized Tripartite Guideline E14. The Clinical Evaluation of QT/QTc Interval Prolongation and Proarrhythmic Potential for Non-Antiarrhythmic Drugs. Available at: http://www.ich. org/cache/compo/276-254-1.html. Accessed December 2012

12. ICH Harmonized Tripartite Guideline E14. ICH E14 Question and Answers, April 2012. Available at: http://www.ich.org/fileadmin/Public_Web_Site/ICH_Products/Guidelines/Efficacy/E14/ E14_Q_As_R1_step4.pdf. Accessed December 2012.

13. Trisenox prescribing information. Availabel at: http://www.accessdata.fda.gov/drugsatfda_docs/label/2010/021248s008s009lbl. pdf. Accessed December 2012.

14. Dennis A, Wang L, Wan X, Ficker E. hERG channel trafficking: Novel targets in drug-induced long QT syndrome. Biochem Soc Trans, 2007; 35: 1060-1063.

15. Dennis AT, Wang L, Wan H, Nassal D, Deschenes I, Ficker E. Molecular determinants of pentamidine-induced hERG trafficking inhibition. Mol Pharmacol, 2012; 81: 198-209.

16. Ficker E, Kuryshev YA, Dennis AT et al. Mechanisms of arsenic-induced prolongation of cardiac repolarization. Mol Pharmacol, 2004; 66: 33-44.

17. Kuryshev YA, Ficker E, Wang L et al. Pentamidine-induced long QT syndrome and block of hERG trafficking. J Pharmacol Exp Ther, 2005; 312: 316-323.

18. Ponte ML, Keller GA, Di GG. Mechanisms of drug induced QT interval prolongation. Curr Drug Saf, 2010; 5: 44-53. 